\title{
Adaptabilidad de Spirulina (Arthrospira) platensis (Cyanophyta) en fotobiorreactor tubular cónico bajo condiciones ambientales
}

\author{
Adaptability of Spirulina (Arthrospira) platensis (Cyanophyta) in \\ conical tubular photobioreactor under environmental conditions
}

\author{
Ronald Huarachi-Olivera ${ }^{1 *}$, Úrsulo Yapo-Pari ${ }^{1}$, Álex Dueñas-Gonza ${ }^{1}$, Rosaura González-Juárez, \\ José Condori-Huamanga², D. G. Pacheco-Salazar ${ }^{2}$, Joe Soto-Flores ${ }^{3}$
}

\begin{abstract}
RESUMEN
Se estudió la adaptabilidad del alga Cyanophyta Spirulina (Arthrospira) platensis (Geitl) cultivada en fotobiorreactor tubular cónico bajo condiciones ambientales con $0,28 \mathrm{~m}^{2}$ en la superficie superior del cono $(0,7 \mathrm{~m}$ de alto $\mathrm{x} 0,6 \mathrm{~m}$ diámetro superior) construido con tubo de PVC (Policloruro de Vinilo) transparente $(12 \mathrm{~mm}$ de diámetro, $34 \mathrm{~m}$ de largo para las 34 espiras que forman el cono y $7 \mathrm{~m}$ para el enfriador), reforzado con una estructura metálica y forrado con hojas de aluminio (volumen total $=5,3 \mathrm{~L}$ ). La superficie interna del cono $\left(0,71 \mathrm{~m}^{2}\right)$ fue expuesta a luz solar con iluminancia de 11.700 lux y radiación solar media igual a $774 \mathrm{~W} / \mathrm{m}^{2} \mathrm{desde}$ el 24 de junio al 12 julio de 2013. La agitación y aireación del cultivo en el fotobiorreactor se realizó con una bomba de aire a un caudal medio de $0,002 \mathrm{~L} / \mathrm{s}$, la misma que dio como resultado una tasa de producción de biomasa seca de $0,298 \mathrm{~g} / \mathrm{m}^{2} /$ día, clorofila"a" de $0,0023 \mathrm{~g} / \mathrm{m}^{2} /$ día y carotenoides de $0,0011 \mathrm{~g} / \mathrm{m}^{2} /$ día. El crecimiento de S. platensis en fotobiorreactor tubular cónico se ajustó a un modelo matemático de crecimiento "Potencial" en 18 días, con estadísticos de ajuste del modelo de $\left(\mathrm{R}^{2}=0,937 ; \mathrm{F}=119,216\right)$.

Palabras clave: fotobiorreactor, iluminancia, UV, Spirulina.
\end{abstract}

\begin{abstract}
We studied the adaptability of the alga Cyanophyta Spirulina (Arthrospira) platensis (Geitl) grown in conical tubular photobioreactor under ambient conditions with $0.28 \mathrm{~m}^{2}$ on the upper surface of the Cone $(0.7 \mathrm{~m} \times 0.6 \mathrm{~m}$ top diameter) constructed with $P V C$ (polyvinyl chloride) pipe transparent (12 mm diameter, $34 \mathrm{~m}$ long for the 34 coils forming the cone and $7 \mathrm{~m}$ for the cooler) reinforced with a metal frame and lined with aluminum foil; (total volume $=5.3 \mathrm{~L})$. The inner surface of the Cone $\left(0.71 \mathrm{~m}^{2}\right)$ was exposed to sunlight with 11700 lux illuminance and solar radiation mean equal to $774 \mathrm{~W} / \mathrm{m}^{2}$ from June 24 to July 12, 2013. Agitation and aeration of the photobioreactor culture was carried out with an air pump to an average flow of $0.002 \mathrm{~L} / \mathrm{s}$ which resulted in a rate of production of dry biomass of $0,298 \mathrm{~g} / \mathrm{m}^{2} /$ day, chlorophyll " $\mathrm{a}$ " $0,0023 \mathrm{~g} / \mathrm{m}^{2} /$ day and carotenoids in $0,0011 \mathrm{~g} / \mathrm{m}^{2} /$ day. The growth of $\mathrm{S}$. platensis in conical tubular photobioreactor was adjusted to a mathematical model of growth "Potential" in 18 days, with statistical adjustment of the model $\left(R^{2}=0.937 ; F=119,216\right)$.
\end{abstract}

Key words: photobioreactor, illuminance, UV, Spirulina.

\section{Introducción}

La fotoadaptación y fotoinhibición ha sido estudiada intensamente en microalgas y plantas superiores, pero principalmente bajo condiciones de laboratorio (Powles, 1984; Oquist, 1987). La microalga Spirulina platensis es una cyanobacteria económicamente importante, producida comercialmente como fuente de alimentación humana, como farmacéuticos, colorantes, polisacáridos y en la industria cosmética. Se han realizado muchos estudios para mejorar las condiciones de crecimiento y cultivo (Ritchmon y Grobbelaar, 1986; Vonshak y Guy, 1992; Torzillo et al., 1998). Sin embargo, pocas publicaciones documentan acerca de los efectos de la radiación ultravioleta (UV) (longitudes de onda

\footnotetext{
1 Laboratorio de Biología Acuática, Departamento Académico de Biología, Universidad Nacional de San Agustín (UNSA), Arequipa, Perú.

2 Departamento Académico de Física, Universidad Nacional de San Agustín (UNSA), Arequipa, Perú.

3 Centro de Microscopia Electrónica, Universidad Nacional de San Agustín (UNSA), Arequipa, Perú.

* Autor para correspondencia: rhuarachi@unsa.edu.pe
}

Fecha de Recepción: 15 Enero, 2014.

Fecha de Aceptación: 19 Noviembre, 2014. 
entre 280-400 nm) sobre este organismo (Wu et al., 2005a; Helbling et al., 2006). En la naturaleza o en estanques operados comercialmente, las células de $S$. platensis son expuestos a radiación solar y son susceptibles a la radiación UV; es conocido que los altos niveles de radiación UV pueden reducir la tasa fotosintética en los ecosistemas acuáticos porque inactivan las enzimas y producen daños en el ADN. Recientemente se ha determinado que los niveles de radiación UV pueden afectar la morfología de las cyanobacterias (Wu et al., 2005a).

Uno de los principales factores limitantes del crecimiento de Spirulina platensis en cultivos bajo condiciones ambientales es la excesiva radiación solar; razón por la que cada vez más se está prestando atención a los niveles crecientes de radiación solar ultravioleta (UV) incidente sobre la superficie terrestre debido al agotamiento del ozono estratosférico. La producción primaria del fitoplancton disminuye por el aumento de la energía solar de longitudes de onda de 290-400 nm (correspondientes a la radiación ultravioleta), en cuyo rango está contenida la radiación ultravioleta tipo A (UVA-320-400 nm) y radiación ultravioleta tipo B (UVB-290-320 nm). La radiación UV también puede afectar la producción fisiológica de comportamiento y biomasa de S. platensis. Sin embargo, poco se ha documentado relativo a este aspecto (Wu et al., 2005b). En el Perú, la Región de Arequipa, de clima desértico por su baja humedad y escasa precipitación, registra una intensidad de radiación solar alrededor de $1.110 \mathrm{~W} / \mathrm{m}^{2}$ durante el verano y $800 \mathrm{~W} / \mathrm{m}^{2}$ durante el invierno (Estación Meteorológica de la escuela de Física-UNSA). La elección de un diseño óptimo de fotobiorreactor para el cultivo masivo de microalgas es un factor importante para la productividad fotosintética general; es así que varios diseños de fotobiorreactores han sido examinados y evaluados en el pasado, en donde los fotobiorreactores cerrados tienen ciertas ventajas frente a los sistemas convencionales de tipo abierto de estanque, actualmente utilizados en cultivo masivo (Watanabe y Hall, 1996). El propósito del presente trabajo fue determinar el efecto de radiación solar sobre el crecimiento de Spirulina platensis en cuanto a su sobrevivencia, por lo tanto, el presente estudio evaluó los efectos de la radiación UV sobre la producción de biomasa y determinación de clorofila-a y carotenoides de $S$. platensis cultivado en fotobiorreactor tubular cónico bajo condiciones ambientales.

\section{Materiales y Métodos}

\section{Material biológico}

La microalga Spirulina platensis usada en este estudio fue obtenida de la Colección de Biotecnología de Microalgas del Laboratorio de Biología AcuáticaUNSA, Arequipa, Perú.

\section{Medio de cultivo}

El cultivo fue mantenido usando la solución hidropónica "La Molina" (Rodríguez-Delfín et al., 2001) al 25\% con la siguiente composición $(\mathrm{g} / \mathrm{L})$ : solución A: superfosfato triple $35 ; \mathrm{KNO}_{3}$ 110; $\mathrm{NH}_{4} \mathrm{NO}_{3} 70$; solución B: $\mathrm{MgSO}_{4} 44$; fetrilón combi 2,5; ácido bórico 0,24 y $\mathrm{NaHCO}_{3} 8 \mathrm{~g}$. Todos los nutrientes fueron disueltos en agua declorada, manteniendo un $\mathrm{pH}=9$ y se autoclavó a $120^{\circ} \mathrm{C}$; $15 \mathrm{Lb}$ de presión por 20 minutos. El volumen de S. platensis que se colocó al fotobiorreactor fue de $4730 \mathrm{~mL}$ manteniendo un $\mathrm{pH}$ entre 9 a 10.

\section{Fotobiorreactor tubular cónico}

El fotobiorreactor utilizado en este estudio consistió de 4 partes: (1) un cono espiral de 34 espiras de $0,7 \mathrm{~m}$ de altura y $0,6 \mathrm{~m}$ de diámetro construido con un tubo transparente de PVC (Policloruro de Vinilo) de $34 \mathrm{~m}$ de longitud y $12 \mathrm{~mm}$ de diámetro interno (2) un enfriador de forma espiral de 11 espiras construido con tubo de PVC (7 m de longitud y $12 \mathrm{~mm}$ de diámetro interno); (3) un desgasificador construido con un recipiente de $1 \mathrm{~L}$ de capacidad; (4) una bomba de aire (modelo ACQ-001; Electromagnetic Air Compress, Boyu) (Figuras 1 y 2) (Watanabe y Hall, 1996). El sistema del fotobiorreactor tubular cónico bajo condiciones ambientales se localizó a $16^{\circ} 24^{\prime} 50^{\prime \prime}$ de Latitud sur y a $71^{\circ} 32^{\prime} 02^{\prime \prime}$ de Longitud oeste con una altitud de $2.344 \mathrm{msnm}$ La superficie externa del cono fue cubierta con hojas de aluminio para aumentar la reflexión de la luz solar el interior y temperatura.

El tubo de PVC transparente fue moldeado formando un espiral cónico, ocupando una superficie de $1,30 \mathrm{~m}^{2}$ y el volumen total del tubo que forma el cono fue de $3,84 \mathrm{~L}$; la relación entre la superficie interior y el volumen total del cono fue de $0,34 \mathrm{~m}^{2} / \mathrm{L}$. El volumen total del sistema, incluyendo el enfriador y tuberías de conexión fue 5,3 L. 


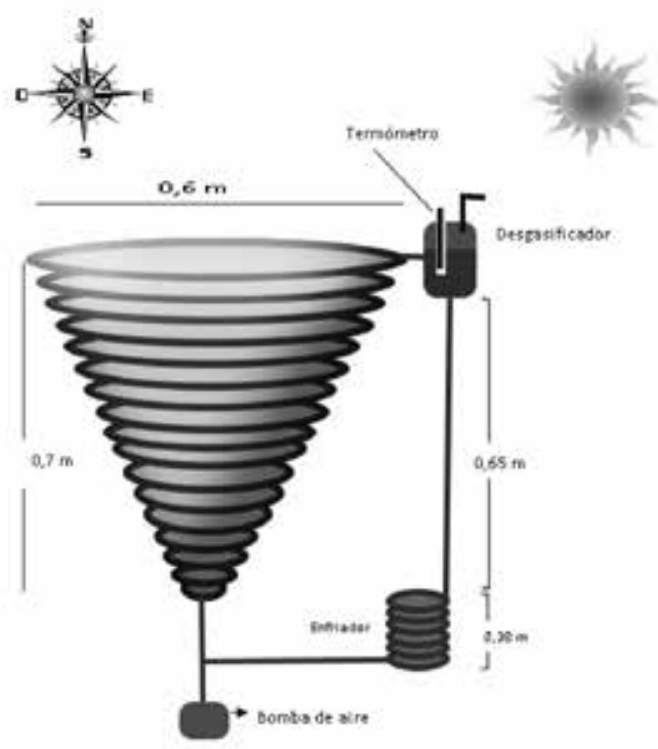

Figura 1. Diagrama esquemático del fotobiorreactor tubular cónico bajo condiciones ambientales para la adaptabilidad de S. platensis. Altura del sistema ( $0,98 \mathrm{~m})$. Altura del cono $(0,7 \mathrm{~m})$. Número de espiras del cono (34). Diámetro del cono $(0,60 \mathrm{~m})$. Número de espiras del enfriador (11). El fotobiorreactor tubular cónico es un sistema construido con manguera PVC (47 m de longitud, $12 \mathrm{~mm}$ de diámetro) utilizando $34 \mathrm{~m}$ de largo para el cono; $7 \mathrm{~m}$ para el enfriador; desgasificador (1L de capacidad con termómetro y tubo de salida de gas). Longitud de la manguera que une el desgasificador con el intercambiador de calor $(0,65 \mathrm{~m})$. El sistema funciona bajo condiciones ambientales $\mathrm{y}$ con una bomba de aire.

\section{Adaptabilidad de $S$. platensis}

El cultivo de $S$. platensis en fotobiorreactor tubular cónico se colocó en el interior de una estructura cúbica forrado con plástico y malla Rachel (50\%) con una iluminancia de 11.700 lux lento (Figuras $2 \mathrm{a}, \mathrm{b}$ ).

\section{Observación ultramicroscópica de los filamentos de Spirulina platensis}

Se realizó en el Centro de Microscopia Electrónica (CME) de la Universidad Nacional de San Agustín (UNSA) de Arequipa, Perú. Para la observación ultramicroscópica de los filamentos de S. platensis se utilizó el microscopio electrónico de barrido marca Philips SEM XL 20 con microanalizador de rayos $\mathrm{X}$ por dispersión de energía marca EDAX DX 4i; las muestras fueron metalizadas con una capa metálica de oro con un

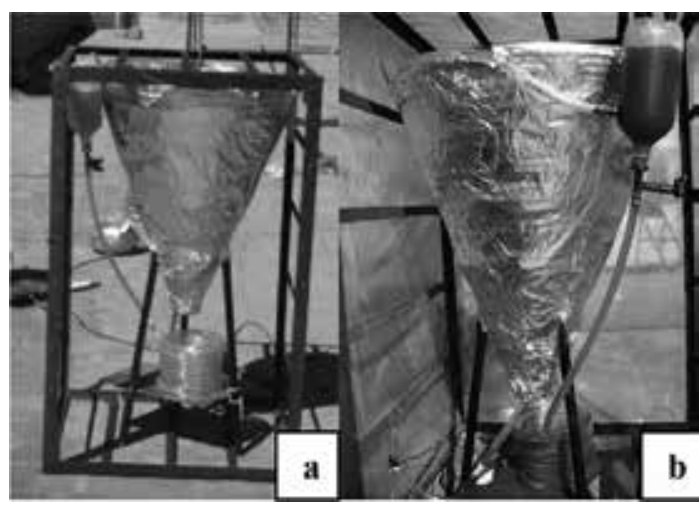

Figura 2. Fotobiorreactor tubular cónico (a) al aire libre (b) en estructura cúbica forrada con plástico y malla Rachel (50\%) en invernadero.

aproximado de $100\left(\mathrm{~A}^{\circ}\right)$ Angstroms de espesor con el metalizador de vacío marca Denton Vacuum Desk II, con la finalidad que sean conductoras y puedan ser analizadas en el microscopio electrónico (Figura 3).

\section{Caudal del Fotobiorreactor Tubular Cónico}

El fotobiorreactor utilizó una bomba de aire, el flujo de líquido fue mediante la inyección de aire (5L/min), para el cálculo del caudal promedio se realizaron diez mediciones entre dos puntos del tubo de PVC según Watanabe y Hall (1996).

\section{Mediciones de temperatura}

Se realizaron mediciones de temperatura en grados Celsius en tres puntos de evaluación; en el invernadero, dentro de la estructura cúbica y en el desgasificador del fotobiorreactor tubular cónico. Las mediciones de temperatura se realizaron tres veces al día a las 8:00 am, 12:00 m y 7:00 pm, tomando las temperaturas máximas y mínimas diarias. Los datos diarios acerca de temperaturas máximas y mínimas se compararon con los registros del Instituto Geofísico de la UNSA (Universidad Nacional de San Agustín).

\section{Medición de iluminancia en fotobiorreactor tubular cónico}

Las mediciones de la iluminación se realizaron entre las 11:00 y 12:00 horas. La iluminación (difusa) medida en interior del fotobiorreactor se realizó utilizando un medidor digital de luz, marca 


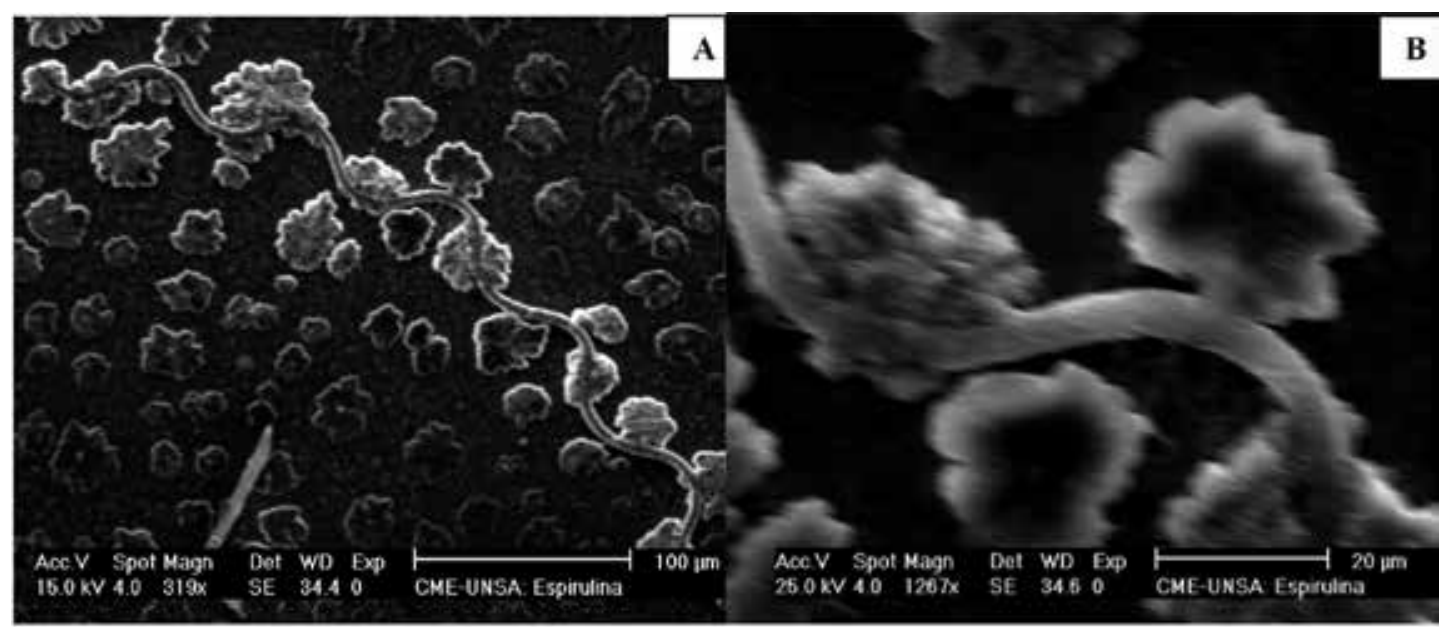

Figura 3. Morfología de Spirulina platensis, micrografía electrónica de los filamentos a diferentes aumentos (A,319X; B,1267X). Fotos tomadas por J. Soto. Centro de Microscopia Electrónica (CME), Universidad Nacional de San Agustín (UNSA), Arequipa, Perú.

EXTECH, Modelo 401025. Se realizaron varias medidas en diferentes posiciones dentro del sistema, donde se verificó que la iluminación disminuye respecto de la distancia del plástico que recubre el fotobiorreactor, dando valores entre 11.700 lux y 114.700 lux.

\section{Determinación espectrofotométrica de la clorofila "a" y carotenoides de $S$. platensis}

Las muestras de producción de S. platensis se concentraron mediante centrifugación (10.000 rpm; $10 \mathrm{~min}$.), decantando el sobrenadante y sometiéndolo a nitrógeno líquido para la rotura de la membrana celular y de esta manera producir la salida del material intracelular, después se resuspendió en un volumen de etanol absoluto hasta cubrir el precipitado, el que se mantuvo en refrigeración a $4^{\circ} \mathrm{C}$ en un frasco ámbar por 2 días. El extracto se centrifugó por 10 min a $8.000 \mathrm{rpm}$ para su clarificación, separando el sobrenadante y anotando el volumen total del extracto. Posteriormente se transfirió $1,5 \mathrm{~mL}$ del extracto clarificado a una cubeta de espectrofotómetro $(1 \mathrm{~cm})$ registrándose la densidad óptica a $750 \mathrm{~nm}$ y $664 \mathrm{~nm}$, enseguida se añadió $0,05 \mathrm{~mL}$ de $\mathrm{HCl} \mathrm{0,1}$ $\mathrm{N}$ agitando con cuidado el extracto acidificado y se tomaron las absorbancias (DO) a $750 \mathrm{~nm}$ y $665 \mathrm{~nm}$; 90 segundos después de la acidificación. Restando las absorbancias que se obtuvieron a $750 \mathrm{~nm}$ (no acidificada y acidificada) a las correspondientes lecturas que se obtuvieron a $664 \mathrm{~nm}$ antes de acidificar y a $665 \mathrm{~nm}$ después de acidificar (la absorbancia registrada a $750 \mathrm{~nm}$ es una medida de la turbidez en el extracto). Utilizando los valores corregidos $(\mathrm{DO}=664 \mathrm{~nm}$ y DO $=665 \mathrm{~nm})$ se calculó la concentración de clorofila-"a" (APHA, 1992). Para la determinación de carotenoides se realizó los métodos anteriores midiendo la densidad óptica a una longitud de onda de $444 \mathrm{~nm}$ en cubetas de vidrio de $1 \mathrm{~cm}$ de paso de luz (Whyte, 1987).

\section{Evaluación del crecimiento de $S$. platensis en fotobiorreactor tubular cónico}

Para evaluar el crecimiento de $S$. platensis se realizó el conteo con cámara de Sedgwick-Rafter y se determinó el número de organismos $/ \mathrm{mL}$. (Figura 4) (Moheimani et al., 2013).

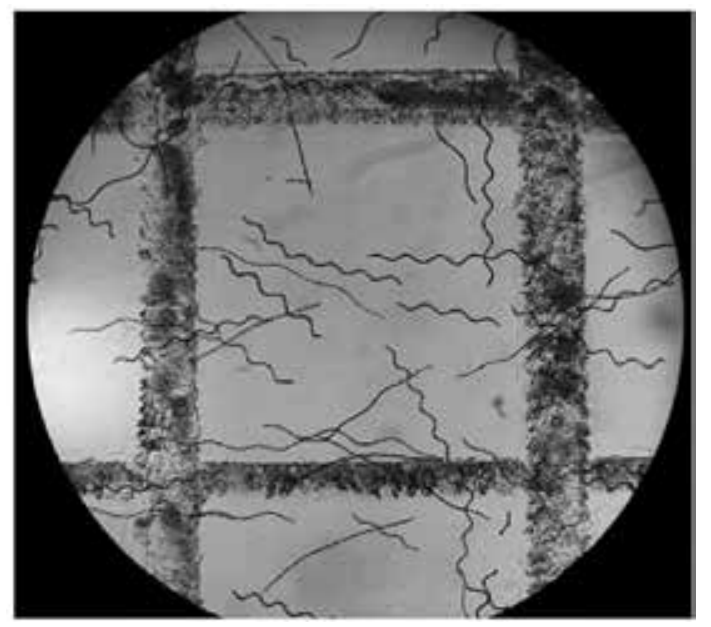

Figura 4. Vista microscópica (40X) de filamentos de Spirulina platensis en cámara de conteo Sedgwick-Rafter. 


\section{Determinación de la biomasa}

Para la determinación de la biomasa seca se procedió a filtrar $350 \mathrm{~mL}$ de muestra de S. platensis, por medio de un papel filtro (marca Whatman y porosidad semilento), luego se secó en estufa durante $2 \mathrm{~h}$ a $105^{\circ} \mathrm{C}$ (Vonshak, 2002).

El otro procedimiento que se utilizó para determinar el crecimiento de $S$. platensis fue por el método del espirúmetro, utilizando un disco Secchi (Planchon y Fuentes, 2009).

\section{Resultados y Discusión}

\section{Cambios morfológicos de filamentos de Spirulina platensis}

En el estudio de la estructura ultramicroscópica de los filamentos de Spirulina platensis mediante microscopia electrónica a diferentes aumentos (319X, 1267X) se observó los extremos romos característicos del filamento de la microalga, mostrando cinco a seis espiras de $60 \mu \mathrm{m}$ aprox., coincidiendo con los caracteres morfológicos típicos de S. platensis (Figura 3) descritos por Fox (1996), describiendo la longitud de espiras entre 30 a $75 \mu \mathrm{m}$.

Como se muestra en la Figura 4 los filamentos de $S$. platensis cultivada en fotobiorreactor tubular cambiaron de una forma espiral típica a una forma recta, por efectos de la radiación ultravioleta (es decir, hubo un menor número de espiras por unidad de longitud del filamento). Según Jeeji y Seshadri (1998), encontraron que cepas de S. platensis con una estructura helicoidal apretada podían tolerar mayor intensidad de luz que las cepas con espirales sueltas. Los filamentos rectos pueden ser transformados a las formas en espiral cuando las células se cambian a condiciones de alta luz y radiación UV directas (Fox, 1996). El cambio en la forma de los filamentos de una espira más relajada a una hélice más apretada, inducidos por la radiación UV o por altos niveles de luz visible, están asociados con la estrategia de protección de esta cyanobacteria para contrarrestar la radiación UV solar al aumentar el autosombreado (Wu et al., 2005a) y por tanto evitar la fotoinhibición. Por el contrario, nuestro estudio muestra que los filamentos de $S$. platensis cambia de una forma espiral a una forma recta en 18 días del cultivo. Este resultado podría sugerir que hay diferentes escalas de adaptación y aclimatación cuando las células reciben la radiación solar total, ya que en escalas cortas de tiempo (es decir, días) los filamentos pueden adquirir formas rectas. Sin embargo, en escalas largas de tiempo (es decir, décadas), la adaptación a la radiación UV solar puede producir cambios en la estructura espiral de una hélice más bien alargada a una hélice muy comprimida (Wu et al., 2005b). Esta forma comprimida de las espiras de $S$. platensis es usualmente observada en estanques de cultivo al aire libre, fuentes naturales como lagos y lagunas donde la incidencia de la luz es menor en comparación a los cultivos en fotobiorreactores tubulares.

En nuestra investigación adicionalmente al cambio en la forma de las espiras de S. platensis en condiciones de radiación directa también se observó variación en la longitud de los filamentos de $S$. platensis, obteniéndose mayor longitud que los alcanzados al inicio del cultivo. Wu et al. (2005b) encontraron que la longitud de los filamentos de $S$. platensis varió de acuerdo con el tratamiento de radiación impuesta a las muestras, porque en la cepa cultivada al aire libre la longitud de los filamentos disminuyó a la mitad del valor inicial después de 3 días de exposición a la radiación UV. El período de adaptación puede variar en las distintas cepas de $S$. platensis, ya que su respuesta a una tensión de luz puede diferir significativamente aunque se hayan cultivado en las mismas condiciones (Vonshak et al., 1996b).

\section{Control de la circulación y la Temperatura}

El medio de cultivo de la producción de $S$. platensis en el tubo de PVC que conecta el desgasificador con el enfriador tuvo una velocidad media de $0,018 \mathrm{~m} / \mathrm{s}$ y un caudal medio igual a $0,002 \mathrm{~L} / \mathrm{s}$. La prueba de comparación ANOVA muestra el estadístico de Fisher $(\mathrm{F}=62,24)$; el mismo que presenta una probabilidad $(\mathrm{P}<0,01)$, demostrando diferencias altamente significativas en los 3 puntos de evaluación de temperatura, como la temperatura en fotobiorreactor, en invernadero (ambiente externo), dentro de estructura cúbica (ambiente interno) (Tabla 1).

La temperatura promedio máxima en 3 puntos de evaluación alcanzada fue de $23,44^{\circ} \mathrm{C}$ a los 3 días y la temperatura media fue de $21,15^{\circ} \mathrm{C}$ hasta los 18 días de evaluación, los límites de control para la temperatura se establecieron en $18,86{ }^{\circ} \mathrm{C}$ como límite inferior y $23,44^{\circ} \mathrm{C}$ como límite superior al 95\% de confianza (Figura 5). 
Tabla 1. Comparación de temperaturas en diferentes puntos de evaluación.

\begin{tabular}{lccccc}
\hline Fuente de variabilidad & Suma de cuadrados & g.l. & Media cuadrática & F & Sig. P \\
\hline Temperatura & 663,186 & 3 & 221,062 & 62,24 & 0,000 \\
Error & 241,509 & 68 & 3,552 & & \\
Total & 904,695 & 71 & & & \\
\hline
\end{tabular}

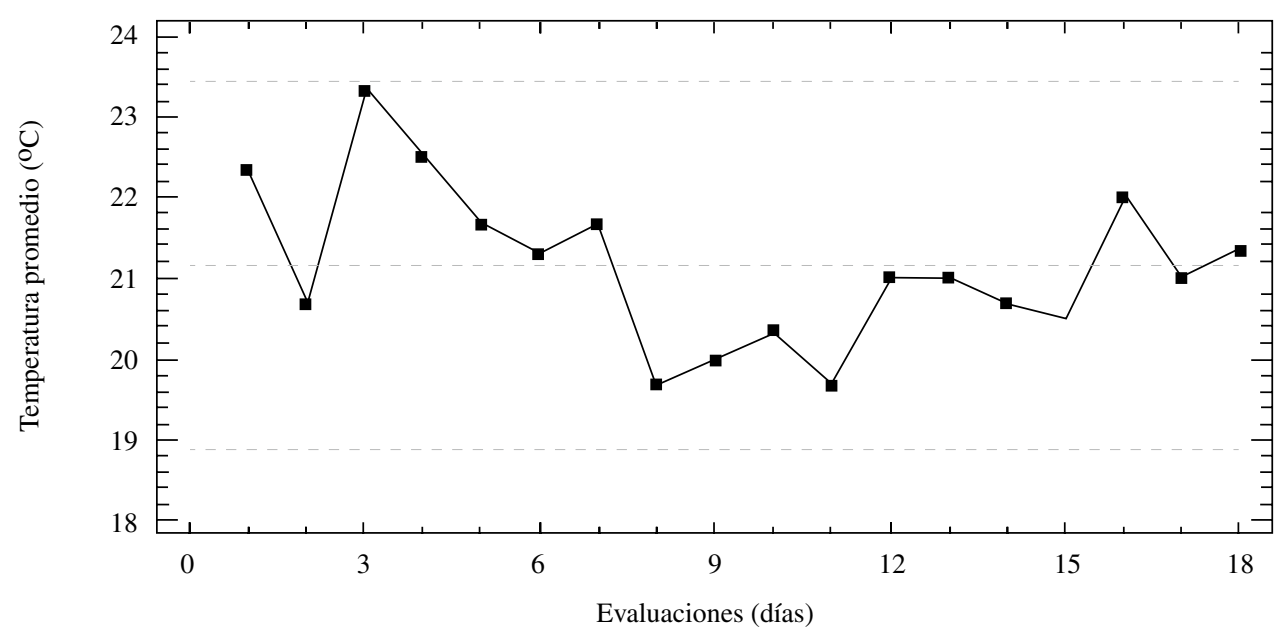

$$
\text { LCS }=23,44 \quad \text { CTR }=21,15 \quad \text { LIC }=18,86
$$

Figura 5. Relación entre la temperatura media de la producción de Spirulina platensis en fotobiorreactor cónico y el tiempo de evaluación (en días). LSC = Límite Superior de Confianza; LIC = Límite Inferior de Confianza; $\mathrm{CTR}=$ Valor medio de la Temperatura.

La prueba de Tukey muestra un mayor registro en la $\mathrm{T}^{\circ}$ (ambiental interna) de $25,38 \pm 3,24^{\circ} \mathrm{C}$; mientras que el menor registro se presentó en la $\mathrm{T}^{\circ}$ (Instituto Geofísico UNSA) de 17,63 $\pm 0,19{ }^{\circ} \mathrm{C}$ (Figura 6). Los resultados obtenidos de temperatura difieren a los encontrados por Watanabe y Hall (1996) sobre su investigación en la producción fotosintética de S. platensis en fotobiorreactor bajo condiciones de laboratorio con iluminación completa alcanzando temperaturas de 35 y $40{ }^{\circ} \mathrm{C}$.

\section{Iluminación y radiación}

Por la situación geográfica de la ciudad de Arequipa, los días son soleados en la mayor parte del año. Las medidas realizadas de la iluminación fueron de 11.700 lux y el ángulo de elevación del sol respecto del plano horizontal superior del fotobiorreactor tubular cónico fue de $56,8^{\circ}$ (en promedio). Estas mediciones se realizaron durante las 11 y 12 horas del día 26 de junio. El experimento se llevó a cabo desde el 24 de junio al 12 de julio de 2013 alcanzando una radiación solar máxima de 774 W/m² según la Estación Meteorológica de la Escuela Profesional de Física (EPF) de la UNSA (Figura 7).

\section{Producción de Biomasa}

De acuerdo con el conteo de filamentos de S. platensis el máximo crecimiento se obtuvo a los 18 días con $142.500 \mathrm{org} / \mathrm{mL}$, cuyo crecimiento se ajusta a un modelo matemático potencial $\left(\mathrm{y}=\mathrm{ax}^{\mathrm{b}}\right.$ $\mathrm{o}$ lny $=\operatorname{lna}+\mathrm{bx})$, mostrando estadísticos de ajuste del modelo $\left(\mathrm{R}^{2}=0,937 ; \mathrm{F}=119,216\right)($ Figura 8$)$ (Guisande et al., 2013).

Se obtuvo una concentración de biomasa de $1,5 \mathrm{~g} / \mathrm{L}$ y productividad de $0,298 \mathrm{~g} / \mathrm{m}^{2} /$ día a 11.700 lux en el interior de fotobiorreactor tubular cónico. La densidad medida mediante disco Secchi $(2,7 \mathrm{~cm})$ fue igual a 0,67 g/L (Planchon y Fuentes, 2009), este muestra que el crecimiento fue menor en comparación 


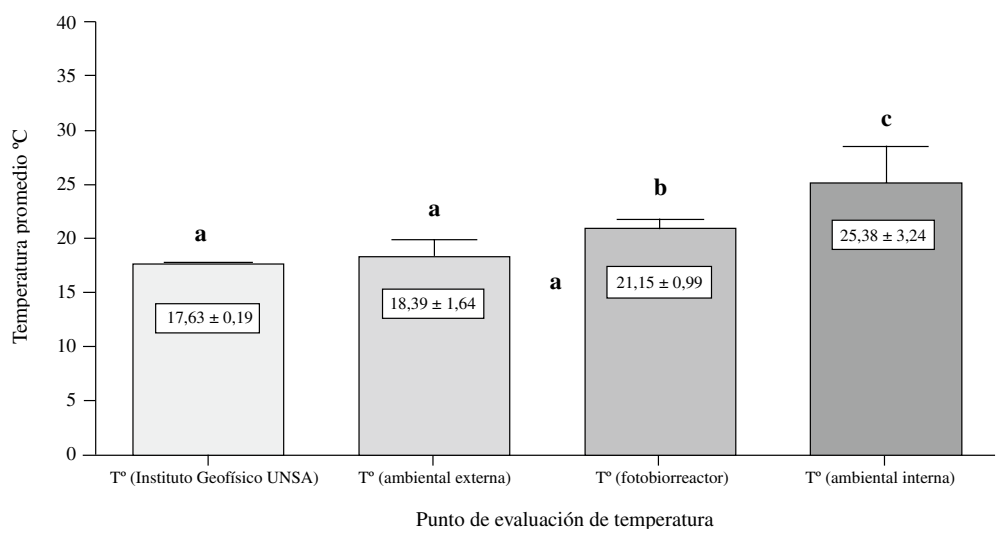

Figura 6. Prueba de comparación múltiple de Tukey para los puntos de evaluación de temperatura.

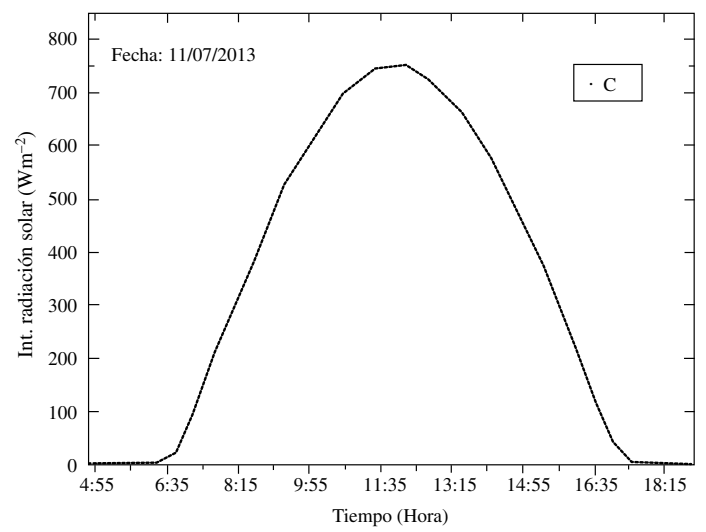

Figura 7. Radiación solar tomada desde las 6:00 h hasta 18:00 h (Fuente: Estación Meteorológica de la Escuela Profesional de Física (EPF) de la UNSA). a la producción de $S$. platensis de $15,9 \mathrm{~g} / \mathrm{m}^{2} /$ día en un cultivo realizado en fotobiorreactor tubular (Watanabe y Hall, 1996), ya que este se realizó en condiciones de laboratorio; además se ha demostrado que la radiación UV inhibe el crecimiento en el cultivo de microalgas, debido al daño causado por la alta densidad del flujo de fotones (Vonshak et al., 1996a). En cultivos realizados al aire libre por Vonshak y Guy (1992) en estanques de $2,5 \mathrm{~m}^{2}$ con una profundidad de $10 \mathrm{~cm}$, agitado con una rueda de paleta a $15 \mathrm{rpm}$, se obtuvo una productividad de $12,7 \pm 0,9 \mathrm{~g} / \mathrm{m}^{2} /$ día a 59.400 lux, siendo mayor en comparación al obtenido a condiciones ambientales y expuestas a la radiación UV. En investigaciones de Spirulina platensis bajo condiciones ambientales controladas en reactores

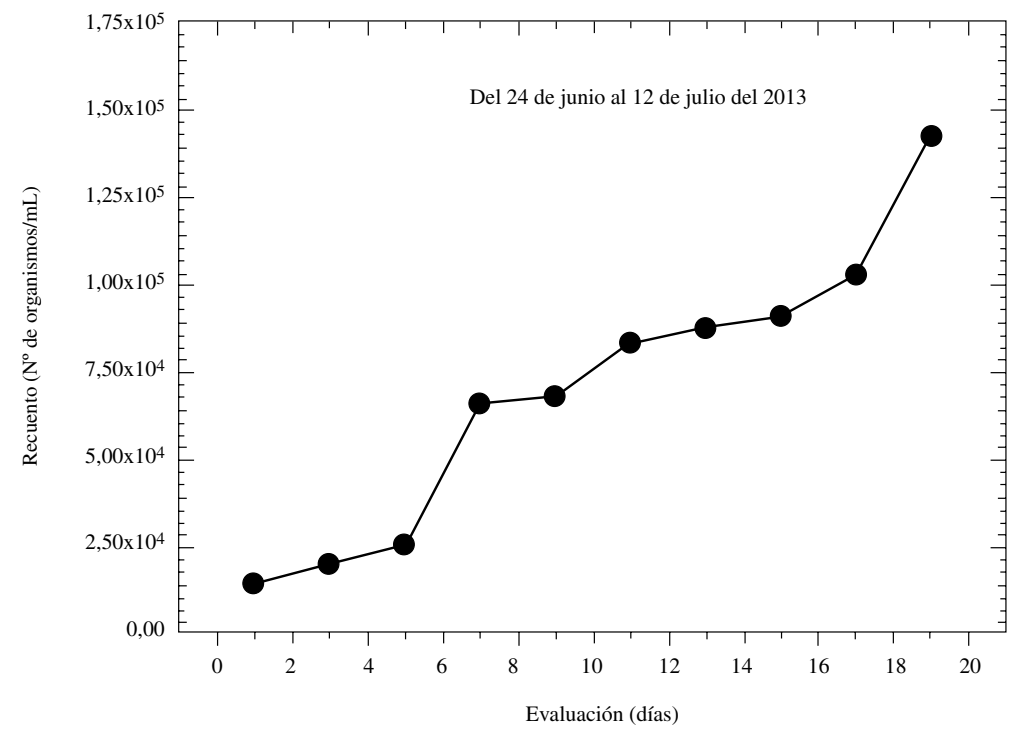

Figura 8. Crecimiento de Spirulina platensis en fotobiorreactor tubular cónico hasta los 20 días de evaluación. 
tubulares se obtuvo concentraciones entre 2 a 2,225 $\mathrm{g} / \mathrm{L}$ de biomasa seca con una irradiación solar total de $254,6 \mathrm{~W} / \mathrm{m}^{2}$ (Vonshak et al., 1996b) y 0,83; 0,44 y $0,61 \mathrm{~g}$ peso seco $\mathrm{L}^{-1} \mathrm{~d}^{-1}$ alcanzados en otoño (septiembre-octubre), invierno (noviembre-diciembre) y marzo, respectivamente (Zittelli et al., 1996).

\section{Concentración de clorofila "a"}

La concentración de clorofila-"a" en valores de biomasa fue de $0,012 \mathrm{~g} / \mathrm{L} \mathrm{o} 0,0023 \mathrm{~g} / \mathrm{m}^{2} /$ día o $0,8 \%$ de la biomasa seca, con una iluminancia de 11.700 lux. La composición de clorofila-“a” presente en Spirulina varía de 0,8 a $1,5 \%$ de materia seca (Paoletti et al., 1980), a comparación de concentración de clorofila entre 0,035 a $0,04 \mathrm{~g} / \mathrm{L}$ en 24 horas en fotobiorreactor tubular (Vonshak et al., 1996b). Los resultados encontrados por Rangel et al. (2004) referentes al crecimiento de $S$. platensis, bajo la influencia de la intensidad de la luz, obtuvieron mayores concentraciones con $0,69 \mathrm{mg} / \mathrm{L} / \mathrm{d}$ de clorofila-"a", pero a una iluminancia más baja con 5.600 lux. La producción de clorofila-“a” por Spirulina platensis en respuesta a diferentes radiaciones obedece a un mecanismo de fotoadaptación en cianobacterias (Post et al., 1985; Rasp et al., 1983), el contenido de clorofila-"a" por célula disminuye cuando se incrementa la intensidad de luz. Similarmente, Utkilen et al. (1983) reporto que el contenido de clorofila por célula se incrementa tres veces en Anacyctis cuando la intensidad de luz fue reducida de 445 a $31 \mu$ mol photon $\mathrm{m}^{-2} \mathrm{~s}^{-1}$.

\section{Concentración de carotenoides}

La concentración de carotenoides totales que se obtuvo fue de $6,118 \mu \mathrm{g} / \mathrm{mL}$ o $0,006 \mathrm{~g} / \mathrm{L}$ o 0,0011 $\mathrm{g} / \mathrm{m}^{2} /$ día o $0,4 \%$ de la biomasa seca. Spirulina platensis posee un porcentaje de carotenoides igual a $0,648 \%$ sobre la base del peso seco de la biomasa (Ramírez y Olvera, 2006). Gao \& Ma (2008) realizaron cultivos al aire libre en tubos de cuarzo para aumentar el ingreso del espectro de la radiación solar obteniendo una concentración menor a la nuestra con $2,5 \mu \mathrm{g} / \mathrm{mg}$ o $0,25 \%$ de biomasa seca. Estos resultados podría deberse a que los carotenoides no son homogéneamente distribuidos en cyanobacterias. El aumento en el contenido de carotenoides (por biomasa y por la clorofila-"a") a niveles más altos de irradiancia se cree que es una adaptación necesaria para proteger las moléculas de clorofila (Goodwin, 1980; Krinsky, 1979; Siefermann-Harms, 1980). Las descripciones en la literatura de contenido de carotenoides varían ampliamente para el género Spirulina (Palla y Busson, 1969; Santillán, 1982; Tanaka et al., 1974). Sin embargo, hay poca información disponible acerca del rango fisiológico de la producción de carotenoides en este organismo y poco se ha hecho en cuanto a la fotoadaptación a largo plazo de Spirulina platensis con diferente distribución espectral y su efecto relativo al contenido de carotenoides.

La Spirulina platensis puede responder a diferentes intensidades y radiación cambiando el contenido de clorofila y carotenoides. Por tanto la calidad y la cantidad de luz son dos posibles variables cuya manipulación podría aumentar la producción total de carotenoides (Gressel, 1979; Rau y Shrott, 1979; Senger, 1982; Senger y Briggs, 1981).

\section{Conclusiones}

Según los resultados obtenidos se demostró que la morfología de Spirulina platensis cambia de una forma espiralada típica a una forma recta por exposición directa a la radiación UV de los rayos solares $774 \mathrm{~W} / \mathrm{m}^{2}$, se obtuvo una biomasa de 1,5 $\mathrm{g} / \mathrm{L}$ y productividad de $0,298 \mathrm{~g} / \mathrm{m}^{2} /$ día a 11.700 lux; clorofila-"a" y carotenoides son tolerantes a la exposición directa de radiación solar, pero con poca cantidad de clorofila-"a" al final del experimento y una cantidad considerable de carotenoides en comparación a otros trabajos, con un crecimiento lento. Las características morfológicas de Spirulina suelen estar relacionados con la calidad del producto. Por lo tanto, la radiación UV solar en cultivos al aire libre influye negativamente en la calidad y el rendimiento de la biomasa cosechada. Por otra parte, un aumento de la radiación UV debido al agotamiento del ozono, podría alterar el desarrollo morfológico de cianobacterias durante la aclimatación o en escalas de tiempo de adaptación. En consecuencia, este efecto sobre las formas, tamaños y contenido de carotenoides afecta los niveles tróficos más altos y las tasas metabólicas, ya que los ecosistemas acuáticos dependen en gran medida de estos parámetros.

\section{Agradecimientos}

A Joseph Le Bour, Tito Cuadros, Percy Apfata y Álex Ticona por la ayuda que nos brindaron en la presente investigación. 


\section{Literatura Citada}

\section{APHA}

1992. Standard Methods for the Examination of Water and Wastewaters. 18va. edición. American Public Health Association. Washington, DC. 1659 p.

Fox, R.

1996. Spirulina: Production \& Potential. Edisud. 232 p.

Gao, K.; Ma, Z.

2008. Photosynthesis and growth of Arthrospira (Spirulina) platensis (Cyanophyta) in response to solar UV radiation, with special reference to its minor variant. Environmental and Experimental Botany. 63: 123-129.

Goodwin, T.W.

1980. Functions of carotenoids. In Goodwin TW (ed.), The Biochemistry of the Carotenoids, Chapter 3.11 p.

Gressel, J.

1979. Blue light photoreception. Photochemistry and Photobiology. 30: 749-754.

Guisande, C.; Vaamonde, A.; Barreiro A.

2013. Tratamiento de datos con R, Statistica y SPSS. $2^{\text {a }}$ Edición electrónica. Ediciones Díaz de Santos. Universidad de Vigo. Galicia-España. 977 p .

Helbling, E. W.; Gao, K.; Ai, H.; Ma, Z.; Villafañe, V. E. 2006. Differential responses of Nostoc sphaeroides and to solar ultraviolet radiation exposure. Journal of Applied Phycology, 18: 57-66.

Jeeji Bai, N.; Seshadri C. V.

1980. On coiling and uncoiling of trichomes in the genus Spirulina. Arch. Hydrobiol., 26: 32-47.

Krinsky, N.I.

1979. Carotenoid protection against oxidation. Pure and Applied Chemistry, 51: 649-660.

Moheimani, N.; Borowitzka, M.; Isdepsky, A.; Sing, F.

2013. Standard Methods for Measuring Growth of Algae and Their Composition. Algae for Biofuels and Energy, Developments in Applied Phycology, 5: 265-284.

Oquist, G.

1987. Environmental stress and photosynthesis. In Progress in Photosynthesis. Research IV (ed. J. Biggins). Martinus Nijhoff Publishers, Dordecht. $10 \mathrm{p}$.

Palla, J.C.; Busson, F.

1969. Étude des carotenoids de Spirulina platensis (Gom.) Geitler (Cyanophycées). C. R. Acad. Sc. Paris., 269: 1704-1707.

Paoletti, C.; Vicenzini, M.; Bocci, F.; Materassi, R.

1980. Composizione biochimica generale delle biomasse di Spirulina platensis e Spirulina maxima (ed.). Prospecttive della coltura di Spirulina in Italia. Roma: Consiglio Nazionale delle Richerche, pp. 111-125.

Planchon, G.; Fuentes, R.

2009. La Spiruline pour tous. Pilgrims Book House. India. $179 \mathrm{p}$.

Post, A.F.; De Wit, R.; Mur, L.R.

1985. Interactions between temperature and light intensity on growth and photosynthesis of the cyanobacterium Oscillatoria agardhii. Journal of Plankton Research, 7: 487-495.

Powles, S.B.

1984. Photoinhibition of photosynthesis induced by visible light. Annual Review of Plant Physiolog,. 35: 15-44.
Ramírez, L.; Olivera, R.

2006. Uso tradicional y actual de Spirulina sp. (Arthrospira sp.). Interciencia, 31: 657-663.

Rangel, C.; Godoy, E.; Monteiro, J.; Sato S.

2004. Chlorophyll production from: Spirulina platensis cultivation with urea addition by fed-batch process. Bioresource Technology, 92: 133-141.

Raps, S.; Wyman, K.; Siegelman, H.W.; Falkowski, P.G.

1983. Adaptation of the cyanobacterium Microcystis aeruginosa to light intensity. Plant Physiology, 72: 829-832.

Rau, W.; Shrott, E.L.

1979. Light mediated biosynthesis in plants. Photochemistry and Photobiology, 30: 755-765.

Richmond, A.; Grobbelaar, J.U.

1986. Factors affecting the output rate of Spirulina platensis with reference to mass cultivation. Biomass, 10:253-264.

Rodríguez-Delfín, A.; Hoyos, M.; Chang, M.

2001. Soluciones Nutritivas en Hidroponía: Formulación y Preparación. CIHNM, UNALM. Lima, Perú. 100 p.

Santillan, C.

1982. Mass production of Spirulina. Experientia, 30: 40-43.

Senger, $\mathrm{H}$.

1982. The effect of blue light on plants and microorganisms. Photochemistry and Photobiolog., 35: 911-920.

Senger, H.; Briggs, W.R.

1981. The blue light receptor(s): Primary reactions and subsequent metabolic changes. Photochemistry and Photobiology. 6: 1-38.

Siefermann-Harms D.

1980. The role of carotenoids in chloroplasts of higher plants. In Mazliak, P.; Beneviste, P.; Costes, C.; Douce, $\mathrm{R}$, (eds,), Biogenesis and function of plant lipids. Elsevier North-Holland Biochemical Press, Amsterdam, 331-340. Chapman and Hall, N.Y., 77-95.

Tanaka, Y.; Matsuguchi, H.; Katayama, T.

1974. Comparative biochemistry of carotenoids in algae-IV. Carotenoids in Cyanophyta, blue-green algae, Spirulina platensis. Mem. Fac. Fish., Kagoshima Univ., 23: 111-115.

Torzillo, G.; Bernardini, P.; Masojidek, J.

1998. On-line monitoring of chlorophyll fluorescence to assess the extent of photoinhibition of photosynthesis induced by high oxygen concentration and low temperature and its effect on the productivity of outdoor cultures of Spirulina platensis (cyanobacteria). Journal of Phycology, 34: 504-510.

Utkilen, H.C.; Briseid, T.; Eriksson, B.

1983. Variation in photosynthetic membrane and pigment content with light intensity for Anacystis nidulans grown in continuous cultures. Journal of General Microbiology, 129: $1717-1720$.

Vonshak, A.

2002. Spirulina platensis (Arthrospira): Physiology, Cellbiology and Biotechnology. Taylor \& Francis. Ben Gurion University of the Negev, Israel. $227 \mathrm{p}$.

Vonshak, A.; Torzillo, G.; Accolla, P.; Tomaselli L. 1996a. Light and oxygen stress in Spirulina platensis (cyanobacteria) grown outdoors in tubular reactors. Physiologia Plantarum, 97: 175-179. 
Vonshak, A.; Chanawongse, L.; Bunnag, B.; Tanticharoen, M. 1996b. Light acclimation and photoinhibition in three Spirulina platensis (cyanobacteria) isolates. Journal of Applied Phycology, 8: 35-40.

Vonshak, A.; Guy, R.

1992. Photoadaptation, photoinhibition and productivity in the blue-green alga, grown outdoors. Plant, Cell \& Environment, 15: 613-616.

Watanabe,Y.; Hall, D.

1996. Photosynthetic production of the filamentous cyanobacterium Spirulina platensis in a cone-shaped helical tubular photobiorreactor. Appl Microbiol Biotechnol., 44: 693-698.

Whyte, J.

1987. Biochemical composition and energy content of six species of phytoplankton used in mariculture of bivalves. Aquaculture, 60: 231-241.
Wu, H.; Gao, K.; Ma, Z.;Watanabe, T.

2005a. Effects of solar ultraviolet radiation on biomass production and pigment contents of Spirulina platensis in commercial operations under sunny and cloudy weather conditions. Fisheries Science., 71: 454-456.

Wu, H.; Gao, K.; Villafañe, V.E.; Watanabe, T.; Helbling, E.W. 2005b. Effects of solar UV radiation on morphology and photosynthesis of filamentous cyanobacterium Arthrospira platensis. Applied and Environmental Microbiology., 71: 5004-5013.

Zittelli, G.; Tomasello, V.; Pinzani, E.; Tredici, M. 1996. Outdoor cultivation of Arthrospira platensis during autumn and winter in temperate climates. Journal of Applied Phycology, 8: 293-301 